\title{
Factors associated with door-in to door-out delays among ST-segment elevation myocardial infarction (STEMI) patients transferred for primary percutaneous coronary intervention: a population-based cohort study in Ontario, Canada
}

Oumin Shi ${ }^{1,2^{*}}$ D, Anam M. Khan ${ }^{2}$, Mohammad R. Rezai ${ }^{2}$, Cynthia A. Jackevicius ${ }^{2,3,5}$, Jafna Cox ${ }^{8}$, Clare L. Atzema ${ }^{2,7}$, Dennis T. Ko ${ }^{2,4,5}$, Thérèse A. Stukel ${ }^{2,5}$, Laurie J. Lambert ${ }^{9}$, Madhu K. Natarajan ${ }^{6}$, Zhi-jie Zheng ${ }^{1}$ and Jack V. Tu²,4,5

\begin{abstract}
Background: Compared to ST-segment elevation myocardial infarction (STEMI) patients who present at centres with catheterization facilities, those transferred for primary percutaneous coronary intervention (PCI) have substantially longer door-in to door-out (DIDO) times, where DIDO is defined as the time interval from arrival at a non-PCI hospital, to transfer to a PCI hospital. We aimed to identify potentially modifiable factors to improve DIDO times in Ontario, Canada and to assess the impact of DIDO times on 30-day mortality.

Methods: A population-based, retrospective cohort study of 966 STEMl patients transferred for primary PCl in Ontario in 2012 was conducted. Baseline factors were examined across timely DIDO status. Multivariate logistic regression was used to examine independent predictors of timely DIDO as well as the association between DIDO times and 30-day mortality.
\end{abstract}

Results: The median DIDO time was 55 min, with $20.1 \%$ of patients achieving the recommended DIDO benchmark of $\leq 30 \mathrm{~min}$. Age $\left(\mathrm{OR}_{>75}\right.$ vs $18-550.30,95 \% \mathrm{Cl}$ : 0.16-0.56), symptom-to-first medical contact $(F M C)$ time $\left(\mathrm{OR}_{61-120 \mathrm{mins}}\right.$ vs < 60mins $0.60,95 \% \mathrm{Cl}: 0.39-0.90 ; \mathrm{OR}_{>120 \mathrm{mins}}$ vs < $60 \mathrm{mins}$ 0.53, 95\% Cl:0.35-0.81) and emergency medical services transport with a pre-hospital electrocardiogram (ECG) (OREMS transport + ECG vs self-transport 2.63, 95\% Cl:1.59-4.35) were the strongest predictors of timely DIDO. Patients with timely ECG were more likely to have recommended DIDO times (33.0\% vs 12.3\%; $P<0.001)$. A significantly higher proportion of those who met the DIDO benchmark had timely FMC-to-balloon times (78.7\% vs $27.4 \% ; P<0.001)$. Compared to patients with DIDO time $\leq 30$ min, those with DIDO times $>90$ min had significantly higher adjusted 30-day mortality rates (OR 2.82, 95\% Cl:1.10-7.19).

\footnotetext{
* Correspondence: somking214@alumni.sjtu.edu.cn

'School of Public Health, Shanghai Jiaotong University School of Medicine,

South Chongqing Road No, Shanghai 227, China

${ }^{2}$ Institute for Clinical Evaluative Sciences, G1 06, 2075 Bayview Avenue,

Toronto, ON, Canada

Full list of author information is available at the end of the article
}

(c) The Author(s). 2018 Open Access This article is distributed under the terms of the Creative Commons Attribution 4.0 International License (http://creativecommons.org/licenses/by/4.0/), which permits unrestricted use, distribution, and reproduction in any medium, provided you give appropriate credit to the original author(s) and the source, provide a link to the Creative Commons license, and indicate if changes were made. The Creative Commons Public Domain Dedication waiver (http://creativecommons.org/publicdomain/zero/1.0/) applies to the data made available in this article, unless otherwise stated. 
(Continued from previous page)

Conclusions: While benchmark DIDO times were still rarely achieved in the province, we identified several potentially modifiable factors in the STEMI system that might be targeted to improve DIDO times. Our findings that patients who received a pre-hospital ECG were still being transferred to non-PCl capable centres suggest strategies addressing this gap may improve patient outcomes.

Keywords: ST-segment elevation myocardial infarction (STEMI), Primary percutaneous coronary intervention (PCI), Door-in to door-out (DIDO), Pre-hospital electrocardiogram (ECG), Mortality

\section{Background}

For patients with ST-Elevation myocardial infarction (STEMI), time-to-treatment is an important modifiable determinant of survival $[1,2]$. Timely primary percutaneous coronary intervention (PCI) is considered the preferred method of reperfusion compared to fibrinolytic therapy, given its lower STEMI mortality rate [3]. However, about $25 \%$ of Canadian residents do not live within a one-hour drive of a PCI-capable centre and often have to be transferred from a non-PCI capable (referral) hospital [4]. Consequent lengthening in receipt of PCI therapy has a demonstrably negative impact on clinical outcomes $[5,6]$.

Delays in providing timely reperfusion therapy can occur at several points along the treatment pathway but take place frequently at the referral hospital [7, 8]. Guidelines for STEMI recommend that the time between arrival to, and transfer from a referral hospital to a PCI-capable hospital, also referred to as the door-in-door-out (DIDO) time, should be $\leq 30 \mathrm{~min}$, and has been widely adopted as an important metric for quality of STEMI care $[1,9]$. However, several studies have noted that this benchmark is rarely achieved [7, 8, 10-12].

In light of this, there is a clear need to develop new strategies or refine pre-existing ones that are responsible for prolonged DIDO times. One such suggestion is having emergency medical services (EMS) personnel administer a 12-lead electrocardiogram (ECG) prior to arrival at the hospital (pre-hospital ECG) for patients suspected of having a STEMI, although the impact on reducing DIDO times has not been well studied [13].

Utilizing a population-based cohort of STEMI patients in Ontario, Canada, we aimed to identify potentially modifiable factors to improve DIDO time. A secondary objective was to examine the impact of DIDO times on 30-day mortality rates adjusted for important confounders. Our findings could have important public health and policy implications, providing 'real world' evidence for areas in the STEMI system that should be targeted for improvement.

\section{Methods}

\section{Data sources}

The Ontario portion of the Canadian Institute for Health Information (CIHI) Discharge Abstract Database (DAD) and National Ambulatory Care Reporting System (NACRS) were used to identify all patients who were hospitalized or presented to an emergency department (ED) for a STEMI event in calendar year 2012. Detailed information on patients was obtained from their medical charts which were abstracted by trained cardiology nurses hired by the research team. Abstracted data included patient characteristics at baseline, key time variables, presenting information and information on the patient transport process. The data were securely transmitted electronically to a database housed at the Institute for Clinical Evaluative Sciences (ICES) in Toronto, Ontario. Given the low-risk nature of this study, Ontario privacy laws allow waivers of informed consent to abstract the data $[14,15]$.

Mortality information was obtained through linkage, utilizing encoded health card numbers, to the Registered Persons Database (RPDB). The RPDB contains socio-demographic and date of death information on all Ontario residents eligible for the Ontario Health Insurance Plan.

\section{Study population}

Figure 1 details the creation of the study cohort. International Classification of Diseases, tenth revision codes (Table S1 in the Data Supplement [see Additional file 1]) were used to identify 6631 patients who had a recorded STEMI event in administrative data. A random sample of $\sim 50 \%$ of the patients in each health region, termed a Local Health Integration Network (LHIN), had their charts abstracted, affording us a representative sample of patients for use in the study. Based on chart reviews, individuals who did not meet the clinical criteria for a STEMI event or were determined to have had an in-hospital STEMI were further excluded. Among 3133 STEMI patients with abstracted charts, we focused on the 1616 patients $(51.6 \%)$ who initially presented to a referral hospital and thus excluded those who were transported directly to a PCI-capable centre or who were discharged home or died in the first hospital. For the purpose of this study, in which timeliness of DIDO for primary PCI was examined, we further excluded those who received fibrinolytic therapy at the first hospital or were missing information on the variables required to 
Patients with a STEMI code in CIHI DAD or NACRS in the

$16 \mathrm{PCl}$-capable hospitals in Ontario in $2012(\mathrm{~N}=6,631)$

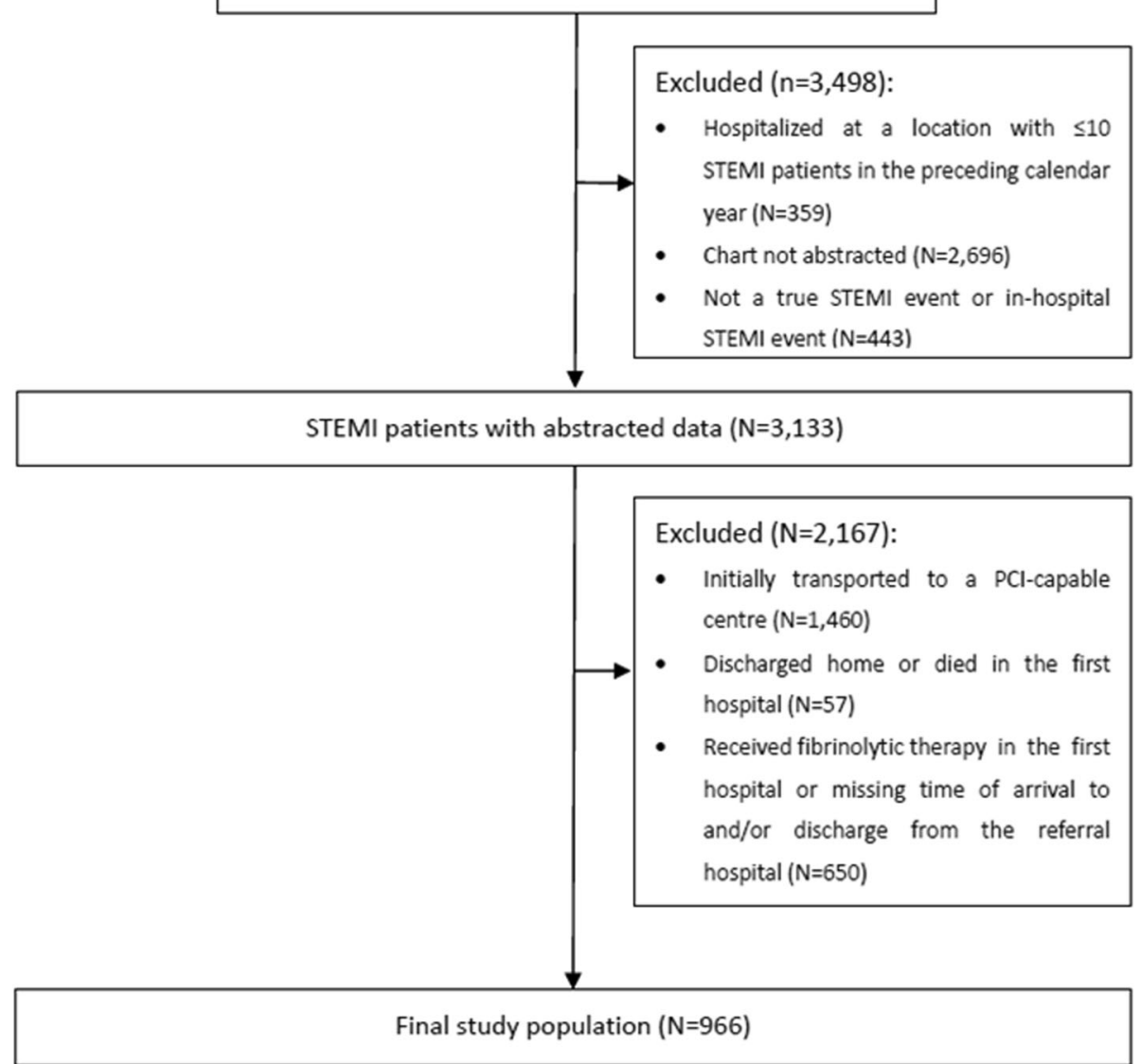

Fig. 1 Cohort creation. CIHI, Canadian Institute for Health Information; DAD, Discharge Abstract Database; NACRS, National Ambulatory Care Reporting System; PCl, percutaneous coronary intervention; STEMI, ST-segment elevation myocardial infarction

compute DIDO time, creating the final study population of 966 patients.

\section{Process of care and clinical outcome definitions}

We assessed several important process of care measures along the STEMI treatment pathway. Where appropriate, benchmarks were selected to be consistent with published Canadian and American guidelines for STEMI care $[1,9,13,16]$. Details on the operationalization of the measures and their associated benchmarks can be found in Table S2 of the Data Supplement [see Additional file 1].

Our primary clinical outcome of interest was all-cause 30-day mortality, defined as death due to any cause within 30 days of the primary PCI procedure.

\section{Statistical analyses}

Baseline patient and process of care measures were examined across timely DIDO status. The proportion of those achieving timely DIDO who also met the benchmark first medical contact (FMC)-to-balloon time of $\leq 120$ min was also examined.

The median durations of process of care indicators comprising the total symptom-to-reperfusion time were compared amongst those who were transported by EMS but did not receive a pre-hospital ECG, transported by EMS and received a pre-hospital ECG and those who transported themselves to hospital.

Generalized estimating equation (GEE) multivariate logistic regression was used to compute odds ratios and corresponding $95 \%$ confidence intervals (CI) to identify baseline characteristics independently associated with a DIDO time $\leq 30$ min [17]. GEE models were used to account for clustering at the LHIN level given that patients residing in the same LHIN may be similarly affected by health system factors which effect STEMI care (e.g., regional STEMI networks) [13]. A similar regression model was used to assess the association between DIDO 
times and 30-day mortality adjusted for patient factors including demographics, traditional cardiac risk factors and co-morbidities, presenting features, symptom-to-FMC time, pre-hospital ECG status and the median durations of several process-of-care measures.

For comparative purposes, we also calculated the crude 30 -day mortality rates amongst the 518 patients who received fibrinolytic therapy at the referral hospital and had data on the times of arrival to the hospital and administration of therapy. This group was not the major focus of this study and as noted earlier, was excluded in the cohort used for the main set of analyses.

Where there was missing data for a variable, it was $<1 \%$ of the sample size and was imputed to the most common value.

All analyses were conducted at ICES in Toronto, Canada using SAS version 9.3 (SAS Institute, Cary NC). This study was approved by the Research Ethics Boards at Sunnybrook Health Sciences Centre and each of the PCI centres in Ontario.

\section{Results}

\section{Patient characteristics and timely DIDO}

The distribution of DIDO times is shown in Figure S1 in the Data Supplement [see Additional file 1]. The median DIDO time was 55 min (interquartile range: 35$112 \mathrm{~min})$. Only 194 patients (20.1\%) achieved the DIDO benchmark, and $\sim 1 / 3$ of patients had DIDO times greater than $90 \mathrm{~min}$.

Fewer patients with DIDO times $\leq 30 \mathrm{~min}$, compared to patients with times $>30 \mathrm{~min}$, were elderly, defined as older than 75 years of age (10.3\% vs $19.9 \%$; adjusted OR [aOR] >75 vs $18-550.30$, 95\% CI: $0.16-0.56$ ) (Table 1 ). Traditional cardiovascular risk factors, co-morbidities and presenting information were not found to be significantly associated with DIDO timeliness.

Approximately $43 \%$ of patients with timely DIDO had acceptable symptom-to- FMC contact times of between 0 and $60 \mathrm{~min}$, in contrast to only $27.5 \%$ of those with untimely DIDO (Table 1). We observed a strong gradient for timeliness of DIDO, such that those who had the longest symptom-to-FMC times had an almost 2-fold decrease in timely DIDO rates compared to those with the shortest time ( $\mathrm{aOR}_{61-120 \mathrm{mins} \text { vs. } 0-60 \mathrm{~min}} 0.60,95 \%$ CI: $0.39-0.90$; $\mathrm{aOR}_{>120 \text { mins vs. } 0-60 \text { min }} 0.53,95 \% \mathrm{CI}$ : 0.35-0.81) (Table 1 ).

\section{Process of care measures}

As depicted in Fig. 2a, receipt of a timely ECG (time to first ECG was less than $10 \mathrm{~min}$ ) was significantly associated with timely DIDO $(P<0.0001)$. Amongst process of care measures, EMS transport with receipt of a pre-hospital ECG was the strongest independent predictor of timely DIDO (aOR EMS + pre-hospital ECG vs self-transport 2.63, 95\% CI: 1.59-4.35) (Table 1; Fig. 2b).
Table 1 Baseline characteristics of the study cohort across Door-in to door-out (DIDO) status, Ontario, Canada, 2012

\begin{tabular}{|c|c|c|c|}
\hline & \multicolumn{2}{|c|}{ DIDO time (mins) } & \multirow{4}{*}{$\begin{array}{l}\text { aOR }(95 \% \mathrm{Cl})^{\mathrm{a}} \\
\text { (timely DIDO } \\
\text { vs. untimely) }\end{array}$} \\
\hline & $\leq 30 \min$ & $>30 \min$ & \\
\hline & $(N=194)$ & $(N=772)$ & \\
\hline & \multicolumn{2}{|c|}{ Frequency (column \%) } & \\
\hline \multicolumn{4}{|l|}{ Age group, years } \\
\hline $18-55$ & $87(44.8)$ & $230(29.8)$ & Ref. \\
\hline $56-65$ & $51(26.3)$ & $234(30.3)$ & $0.57(0.39-0.87)$ \\
\hline $66-75$ & $36(18.6)$ & $154(19.9)$ & $0.61(0.37-0.99)$ \\
\hline$>75$ & $20(10.3)$ & $154(19.9)$ & $0.30(0.16-0.56)$ \\
\hline Sex, females & $33(17.0)$ & $194(25.1)$ & $0.72(0.46-1.15)$ \\
\hline \multicolumn{4}{|c|}{ Cardiovascular risk factors } \\
\hline Diabetes mellitus & $39(20.1)$ & $167(21.6)$ & $0.95(0.62-1.45)$ \\
\hline Current smoker & $88(45.4)$ & $302(39.1)$ & $0.96(0.67-1.39)$ \\
\hline Hypertension & $93(47.9)$ & $390(50.5)$ & $0.98(0.68-1.41)$ \\
\hline
\end{tabular}

Previous cardiovascular clinical events

$\begin{array}{llll}\text { Myocardial infarction } & 21(10.8) & 99(12.8) & 0.68(0.70-3.03) \\ \text { Angina } & 8(4.1) & 41(5.3) & 0.93(0.39-2.22) \\ \text { COPD } & 7(3.6) & 38(4.9) & 0.94(0.38-2.33) \\ \text { Stroke } & 7(3.6) & 30(3.9) & 1.43(0.58-3.57)\end{array}$

Presenting characteristics

$\begin{array}{llll}\text { Cardiac arrest at scene } & 13(6.7) & 51(6.6) & 0.69(0.71-2.04) \\ \text { Elevated cardiac enzymes }^{c} & 171(88.1) & 675(87.4) & 1.22(0.71-2.04) \\ \text { Off-hours presentation }^{d} & 122(62.9) & 508(65.8) & 0.91(0.64-1.28)\end{array}$

Symptom to FMC time, mins

$\begin{array}{llll}0-60 & 84(43.3) & 212(27.5) & \text { Ref. } \\ 61-120 & 56(28.9) & 268(34.7) & 0.60(0.39-0.90) \\ >120 & 54(27.8) & 292(37.8) & 0.53(0.35-0.81) \\ \text { ransport to first hospital } & & & \\ \text { Self-transport } & 103(53.1) & 510(66.1) & \text { Ref. } \\ \text { EMS transport with ECG } & 36(18.6) & 71(9.2) & 2.63(1.59-4.35) \\ \text { EMS transport without ECG } & 55(28.4) & 191(24.7) & 1.45(0.95-2.22)\end{array}$

Abbreviations: $a O R$ adjusted odds ratio, $C I$ confidence interval, $C O P D$ chronic obstructive pulmonary disease, DIDO door-in to door-out, ECG electrocardiogram, EMS emergency medical services, FMC first medical contact, mins minutes, Ref reference

aLogistic regression model fully adjusted for all the variables in the table ${ }^{\mathrm{b}}$ Door-in to door-out times were considered timely if they were $\leq 30 \mathrm{~min}$ ${ }^{\mathrm{C}}$ Elevated cardiac enzyme levels were defined as having at least one of the following occur within the first $24 \mathrm{~h}$ of the first medical contact: 1) a rise in troponin levels above the upper reference limit or the level indicative of acute myocardial infarction, or 2) a rise in creatine kinase $\mathrm{MB}$ or creatine kinase more than twice the upper limit of normal as defined on the lab report ${ }^{\mathrm{d}}$ Defined as presentation to a hospital before $9 \mathrm{am}$ or after $5 \mathrm{pm}$ on weekdays and anytime on weekends

\section{Timely reperfusion}

A significantly $(P<0.0001)$ higher proportion of those who met the DIDO benchmark also had timely FMC-to-balloon times, with rates almost three times higher in the former group (78.7\% vs 27.4\%) (Fig. 2c). 

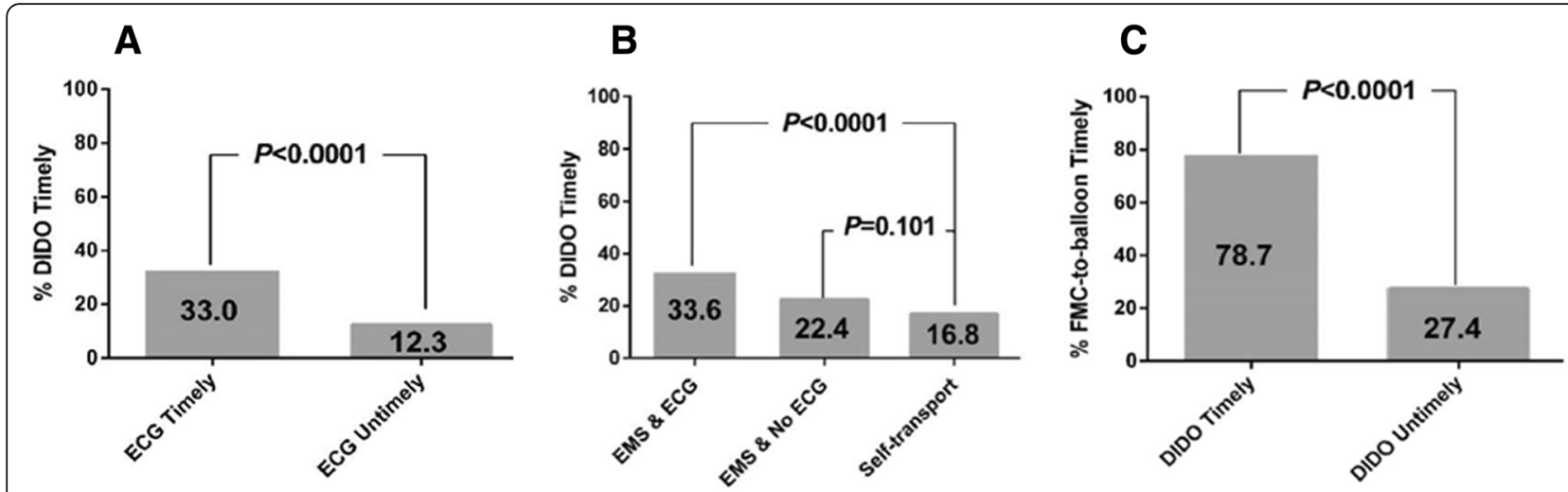

Fig. 2 Prevalence of door-in to door-out times of $\leq 30 \mathrm{~min}$ (timely) across timely electrocardiogram ( $\leq 10 \mathrm{~min}$ ) status (a), and hospital transport groups (b). Percentage of patients who achieved the first medical contact-to-balloon benchmark of $\leq 120$ min across timely door-in to door-out status (c). DIDO, door-in to door-out; ECG, electrocardiogram; EMS, emergency medical services; FMC, first medical contact

Overall, $47.4 \%$ of the transferred patients in our study met the FMC-to-balloon time benchmark.

The breakdown of the components of median reperfusion time across those who self-transported to hospital, were transported by EMS but did not receive a pre-hospital ECG, and those who were transported by EMS and had a pre-hospital ECG are shown in Fig. 3. The median times were 225, 216 and $199 \mathrm{~min}$, respectively. Symptom-to-door time accounted for the greatest duration of median reperfusion time, followed by DIDO times, which were significantly shorter amongst those who were transported by EMS and received pre-hospital ECG compared to the self-transport group (Symptom-to-door time: $85 \mathrm{~min}$ vs $106 \mathrm{~min}$, DIDO: $47 \mathrm{~min}$ vs 56 min; $P<0.05$ ). $33.6 \%$ of those in the former group had timely DIDO compared to only $22.4 \%$ and $16.8 \%$ in the EMS and no pre-hospital ECG group and self-transport groups, respectively (Fig. 2B).

\section{0-day mortality}

Table 2 shows 30 -day mortality rates across DIDO times. Crude 30 -day mortality rates ranged from $4.1 \%$ amongst those with DIDO times of $\leq 30 \mathrm{~min}$ to $11.6 \%$ in those with DIDO times in excess of $90 \mathrm{~min}$. While only patients with DIDO times $>90$ min had significantly higher mortality rates (aOR 2.82, 95\% CI: 1.10-7.19) a mortality gradient was evident.

Patients who received fibrinolytic therapy in the first hospital were excluded from the main analyses, but their crude 30-day mortality rate $(6.6 \%)$ did not differ

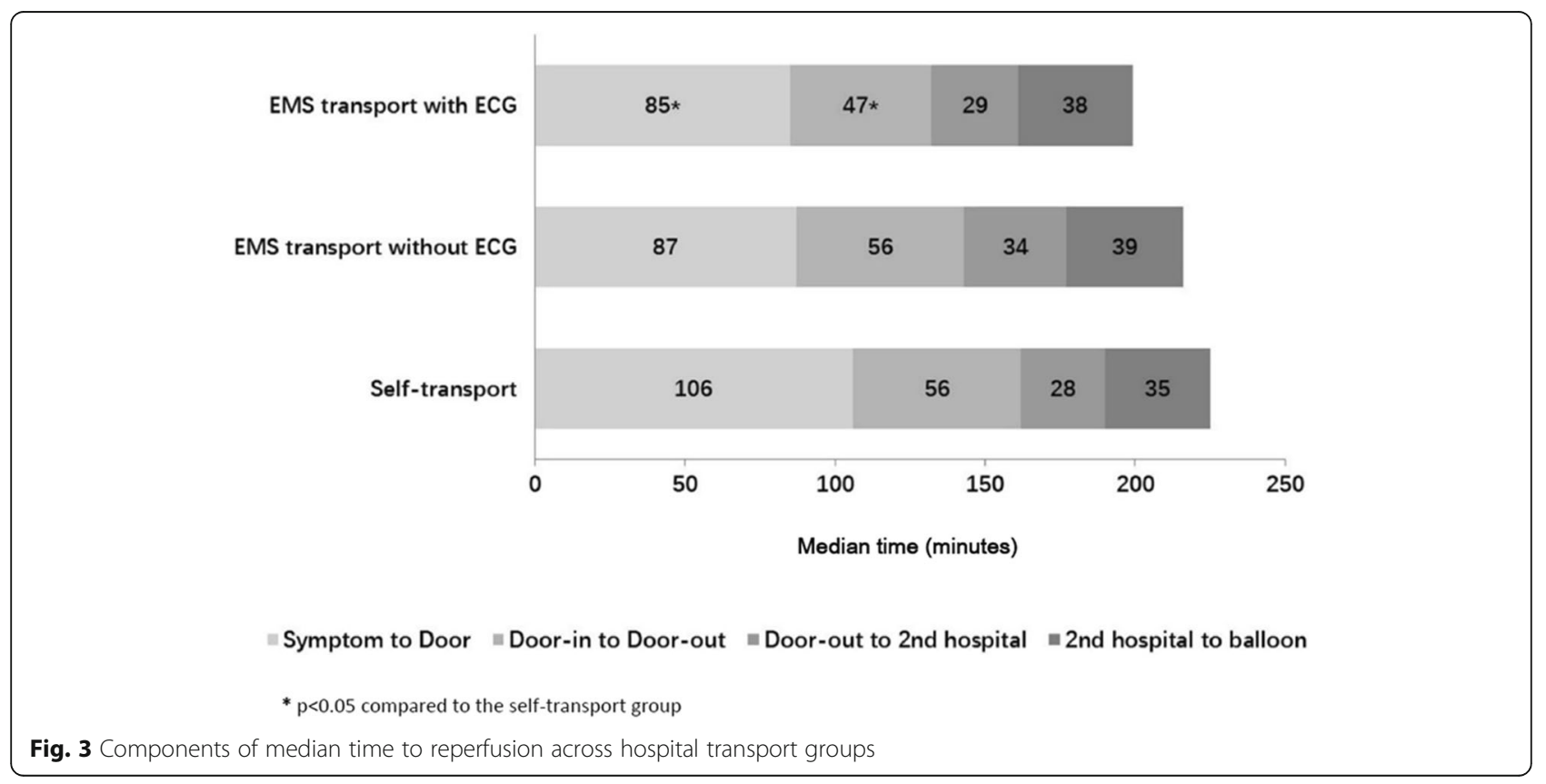


Table 2 Association between door-in to door-out times and 30-day all-cause mortality amongst patients transferred for primary percutaneous coronary intervention, Ontario, Canada, 2012

\begin{tabular}{llll}
\hline & Number of events / Patient population & Crude 30-day mortality rate (\%) & aOR (95\% Cl) \\
\hline DIDO time (mins) & & & Ref. \\
$\leq 30$ (timely) & $8 / 194$ & 4.1 & $1.05(0.38-2.89)$ \\
$31-60$ & $17 / 333$ & 5.1 & $1.73(0.58-5.09)$ \\
$61-90$ & $12 / 145$ & 8.3 & $2.82(1.10-7.19)$ \\
$>90$ & $34 / 294$ & 11.6 & N/A \\
Overall & $71 / 966$ & 7.3 & \\
\hline
\end{tabular}

Abbreviations: $a O R$ adjusted odds ratios, $\mathrm{Cl}$ confidence intervals, DIDO door-in to door-out, mins minutes, $N / A$ not applicable, Ref reference aLogistic regression model was adjusted for patient demographics, traditional cardiac risk factors and co-morbidities, presenting features, symptom-to-FMC time, pre-hospital ECG status and times for process-of-care measures

significantly from patients transferred for primary PCI (7.3\%; $P=0.676)$. Crude mortality rates for those administered fibrinolytic therapy can be found in Table S3 in the Data Supplement [see Additional file 1]. Patients with DIDO times $>90 \mathrm{~min}$ had significantly higher crude mortality rates than those who got timely fibrinolytic therapy $(11.6 \%$ vs. $5.5 \% ; P=0.012)$.

\section{Discussion}

In the present study, we utilized a population-based cohort of STEMI patients in Ontario, Canada to identify potentially modifiable factors that can improve DIDO times and the impact of DIDO times on long-term mortality. Only $20 \%$ of STEMI patients who were transferred from a referral hospital to a PCI-capable centre for primary PCI met the DIDO time of $\leq 30 \mathrm{~min}$. Age was the strongest patient-level predictor of DIDO times. Transport to hospital by EMS in combination with receiving a pre-hospital ECG was the strongest process of care measure associated with DIDO times. In particular, this group had substantively shorter symptom to reperfusion times compared to those who transported themselves to hospital, driven largely by significantly shorter symptom to door and DIDO times. The mortality gradient for DIDO times observed in our study is important as it demonstrates poor long-term outcomes for those who experienced longer DIDO times. The crude 30-day mortality rates in patients transferred for primary PCI was higher than those who got fibrinolytic therapy, in particular for those with DIDO times over $90 \mathrm{~min}$.

Our finding that achievement of benchmark DIDO time was low $(20 \%)$ is consistent with previous studies $[7,8,10-12]$. A study using national registry data from the United States reported that only $11 \%$ of patients achieved DIDO benchmarks, and other studies reported rates of $\sim 10 \%[8,10,11]$. Canadian studies have reported higher rates than American-based studies, one reported 14\% in Quebec [7, 10]. Several factors could account for the disparities in rates including varying characteristics of the study population, regional differences in policies and design of the STEMI system and/or changes in trends over time [7, 8, 10-12]. The latter may be especially pertinent in this case given that our work is more recent than other cited studies.

The guidelines indicate that $90 \%$ of patients transferred from a referring hospital should meet the 120-min time-to-treatment standard [1]. Less than 50\% of transferred patients in our study met the FMC-to-balloon time benchmark. We observed stark differences in achievement of FMC-to-balloon time benchmark across DIDO status. Most transferred patients with DIDO time $>30 \mathrm{~min}$ do not have absolute contraindications for fibrinolytic therapy $[18,19]$. In our and previous studies, about half of transferred patients had a DIDO time $>60 \mathrm{~min}$, with few of these individuals receiving timely PCI $[7,8,10-12]$. Delayed transfer of STEMI patients with DIDO time $>60$ min may not be a superior strategy to the timely use of fibrinolytic therapy $[1,3,20]$. One potential option to address prolonged DIDO times might be to increase the use of fibrinolytic therapy at referral hospitals, followed by transfer for catheterization post-lytic therapy [21].

Elderly age was found to be one of the strongest predictors of DIDO time in our study which is in light with previous studies [7, 10]. Elderly individuals may be less likely to present with classic STEMI symptoms (e.g., retrosternal chest pain) and/or more likely to have ECGs which are complex to interpret, that may delay diagnosis and recognition of a STEMI. Our findings highlight there is a need to bring awareness to the complexity and non-traditional nature of the symptoms with which these elderly patients sometimes present in order to improve DIDO times for this group. Prior studies have shown the disparities in time to reperfusion for STEMI patients in the elderly $[22,23]$. Better software that can accurately read difficult ECGs, enhanced training for medical personnel and public awareness campaigns might improve DIDO times.

We observed that patients transported by EMS personnel, whether in association with a pre-hospital ECG or not, resulted in shorter symptom-to-door times compared to those self-transported patients. However, 
DIDO times were found to be significantly shorter compared to patients who self-transported only amongst those who received a pre-hospital ECG, suggesting that while EMS transport, irrespective of pre-hospital ECG status affects symptom-to-door time, for DIDO times it is the receipt of pre-hospital ECG that makes a difference. Provision of a pre-hospital ECG was not a universally adopted or mandated practice in Ontario in 2012 [9]. However, the findings of the present study suggest that policies which mandate and fund equipping ambulances with this technology and training EMS personnel to administer these ECG's might have a significant impact on reduction of DIDO times and mortality.

Ideally, provision of a pre-hospital ECG should allow for a quicker diagnosis, thus facilitating the activation of STEMI protocols, result in more direct transfers to PCI-capable centres or mitigate delays in the referral ED [24]. Notably, our study found that a fair proportion (11.1\%) of individuals received a pre-hospital ECG and were still initially transferred to a non-PCI capable hospital. Contributing factors may include diagnostic uncertainty, incorrect interpretation of the ECG and/or a clinically unstable patient. It is also plausible that some ambulances continue to bring patients to the nearest community hospital, regardless of ECG findings because the PCI-capable centre is further away, which would identify an area to target for improvement. Increased clarity in the guidelines around when EMS personnel should bypass the closest centre in favor of a more distant PCI-capable hospital, which is integrated within a STEMI protocol, may in part help alleviate this problem. In 2015, a STEMI bypass protocol in Ontario enables paramedics to bypass local hospitals and transport patients with STEMI directly to PCI-capable centres if arrival at the PCI centre will be $\leq 60 \mathrm{~min}$ from the first medical contact [9]. These guidelines came into effect in the province in 2017 and future studies should examine whether new guidelines have reduced the number of patients with STEMI-positive ECG's being transferred to non-PCI capable hospitals. Our findings show that the major delay in reperfusion times occurs at the referral hospital, in line with previous studies' findings [10, 25]. However, it should also be recognized that it may be difficult in EDs to provide care within guideline recommendations for every patient, especially for those with atypical presentations.

The present study aids in generalizability of findings to other populations. The fact that we examined important process of care measures including receipt of pre-hospital ECG's, which have not been extensively studied enabled us to assess the impact of current STEMI management recommendations and guidelines on DIDO times so that we can ascertain a more complete picture of the STEMI care system and identify areas for improvement. Additionally, the present study found DIDO times to be an independent predictor of 30-day mortality, with the observed gradient strengthening the conclusion, and suggests the adverse impact of long DIDO times extends beyond the hospital visit itself. Previous studies have observed similar gradients for in-hospital mortality only or have been underpowered to make inferences regarding longer-term mortality outcomes adjusted for important confounders [10].

However, the present study presents with some important limitations. First, information on key time intervals was ascertained through retrospective chart review and recorded times could not be independently validated. Secondly, we were unable to collect information on the distance to the referral hospital or between the referral hospital and the PCI-capable centre or the details of patient symptoms as they were not available or well documented. Lastly, the administrative STEMI codes we used have not been validated. However, they were applied consistently across all records and those who were identified as STEMI cases from administrative data had their diagnosis verified via chart review by a trained cardiology research nurse, making it unlikely that non-STEMI cases were included in the study.

\section{Conclusions}

In conclusion, our findings suggest that benchmark DIDO times were not achieved for a majority of transferred STEMI patients in the province. Patient age and process of care measures, namely symptom-to-FMC time and receipt of a pre-hospital ECG, were identified to be strong independent predictors of DIDO time. Despite provision of pre-hospital ECGs, a fair proportion of patients were still transferred to non-PCI capable hospitals, suggesting that policies and system-level changes aimed at bypassing non-PCI capable hospitals in favor of PCI-capable hospitals for those with STEMI positive ECGs could have an important impact on outcomes.

\section{Additional file}

Additional file 1: Data supplement containing additional tables and figures (e.g., disease classification codes, benchmark times etc.). (DOCX 130 kb)

\section{Abbreviations}

AOR: Adjusted odds ratio; Cl: Confidence interval; $\mathrm{ClHI}$ : Canadian Institute for Health Information; COPD: Chronic obstructive pulmonary disease;

D2B: Door-to-balloon time; DAD: Discharge Abstract Database; DIDO: Door-in to door-out; ECG: Electrocardiogram; EMS: Emergency medical services;

FMC: First medical contact; mins: Minutes; NACRS: National Ambulatory Care Reporting System; PCI: Percutaneous coronary intervention; Ref: Reference; STEMI: ST-segment elevation myocardial infarction; TRANSFER-AMI: Trial of Routine Angioplasty and Stenting after Fibrinolysis to Enhance Reperfusion in Acute Myocardial Infarction 


\section{Acknowledgements}

Parts of this material are based on data and/or information compiled and provided by the Canadian Institute for Health Information (CIHI). The analyses, conclusions, opinions, and statements expressed in the material are those of the authors and not necessarily those of $\mathrm{CIHI}$. This study was supported by the Institute for Clinical Evaluative Sciences (ICES), which is funded by an annual grant from the Ontario Ministry of Health and LongTerm Care (MOHLTC). The opinions, results and conclusions reported in this paper are those of the authors and are independent from the funding sources. No endorsement by ICES or the Ontario MOHLTC is intended or should be inferred.

\section{Funding}

Supported by operating grants from the Institute of Circulatory and Respiratory Health (ICRH)- Canadian Institutes of Health Research (CIHR) Chronic Diseases Team (grant no. TCA 118349 and grant no. FRN 111035). Dr. Tu is supported by a Tier 1 Canada Research Chair in Health Services Research and an Eaton Scholar award from the Department of Medicine, University of Toronto.

\section{Availability of data and materials}

The data set from this study is held securely in coded form at the Institute for Clinical Evaluative Sciences (ICES). While data sharing agreements prohibit ICES from making the data set publicly available, access may be granted to those who meet pre-specified criteria for confidential access, available at www.ices.on.ca/DAS.

\section{Authors' contributions}

JVT was involved in obtaining data used for this study. JVT, CAJ, JC, CLA and DTK designed the study. OS and MRR analyzed the data. OS, AMK, CAJ, JC, CLA, DTK, TAS, LL, MKN, ZZ and JVT interpreted the study findings. OS, JVT and AMK drafted the manuscript. All authors read and approved the final manuscript.

\section{Ethics approval and consent to participate}

This study was approved by the Research Ethics Boards at Sunnybrook Health Sciences Centre and each of the 16 Percutaneous Coronary Intervention (PCl) centres in Ontario, Canada.

Given the low-risk nature of this study, Ontario privacy laws allow waivers of informed consent to abstract the data.

\section{Consent for publication}

Not applicable.

\section{Competing interests}

The authors declare that they have no competing interests.

\section{Publisher's Note}

Springer Nature remains neutral with regard to jurisdictional claims in published maps and institutional affiliations.

\section{Author details \\ 'School of Public Health, Shanghai Jiaotong University School of Medicine, South Chongqing Road No, Shanghai 227, China. Institute for Clinical Evaluative Sciences, G1 06, 2075 Bayview Avenue, Toronto, ON, Canada. ${ }^{3}$ Western University of Health Sciences, 309 E 2nd St, Pomona, California, USA. ${ }^{4}$ Schulich Heart Centre, Sunnybrook Health Sciences Centre, 2075 Bayview Avenue, Toronto, ON, Canada. ${ }^{5}$ University of Toronto, 27 King's College Circle, Toronto, ON, Canada. ${ }^{6}$ Department of Medicine, Hamilton Health Sciences, McMaster University, 1200 Main St W, Hamilton, ON, Canada. ${ }^{7}$ Sunnybrook Health Sciences Centre, 2075 Bayview Avenue, Toronto, ON, Canada. ${ }^{8}$ Dalhousie University, 6299 South St, Halifax, NS, Canada. ${ }^{9}$ Cardiology Evaluation Unit, Institut national d'excellence en santé et en services sociaux (INESSS), 2021, Avenue Union, Bureau 10.083, Montréal, Québec, Canada.}

\section{Received: 16 January 2018 Accepted: 16 October 2018}

\section{Published online: 29 October 2018}

\section{References}

1. O'Gara PT, Kushner FG, Ascheim DD, Casey DE, Chung MK, de Lemos JA, et al. 2013 ACCF/AHA guideline for the management of ST-elevation myocardial infarction: a report of the American College of Cardiology
Foundation/American Heart Association task force on practice guidelines. Circulation. 2013:127(4):e362-425.

2. Rathore SS, Curtis JP, Chen J, Wang Y, Nallamothu BK, Epstein AJ, et al. Association of door-to-balloon time and mortality in patients admitted to hospital with ST elevation myocardial infarction: national cohort study. BMJ. 2009:338:b1807.

3. Keeley EC, Boura JA, Grines CL. Primary angioplasty versus intravenous thrombolytic therapy for acute myocardial infarction: a quantitative review of 23 randomised trials. Lancet. 2003;361(9351):13-20.

4. Hameed SM, Schuurman N, Razek T, Boone D, Van Heest R, Taulu T, et al. Access to trauma Systems in Canada. J Trauma. 2010;69(6):1350-61.

5. Jollis JG, Roettig ML, Aluko AO, Anstrom K, Applegate RJ, Babb JD, et al. Implementation of a statewide system for coronary reperfusion for STsegment elevation myocardial infarction. JAMA. 2007;298(20):2371-80.

6. Lambert L, Brown K, Segal E, Brophy J, Rodes-Cabau J, Bogaty P. Association between timeliness of reperfusion therapy and clinical outcomes in STelevation myocardial infarction. JAMA. 2010;303(21):2148-55.

7. Lambert LJ, Brown KA, Boothroyd L, Segal E, Maire S, Kouz S, et al. Transfer of patients with ST-elevation myocardial infarction for primary percutaneous coronary intervention: a province-wide evaluation of "door-in to door-out" delays at the first hospital. Circulation. 2014;129(25):2653-60.

8. Miedema MD, Newell MC, Duval S, Garberich RF, Handran CB, Larson DM, et al. Causes of delay and associated mortality in patients transferred with STsegment-elevation myocardial infarction. Circulation. 2011;124(15):1636-44.

9. CCN. Recommendations for Best Practice STEMI Management in Ontario. 2013.

10. Wang TY, Nallamothu BK, Krumholz HM, Li S, Roe MT, Jollis JG, et al. Association of door-in to door-out time with reperfusion delays and outcomes among patients transferred for primary percutaneous coronary intervention. JAMA. 2011;305(24):2540-7.

11. Herrin J, Miller LE, Turkmani DF, Nsa W, Drye EE, Bernheim SM, et al National performance on door-in to door-out time among patients transferred for primary percutaneous coronary intervention. Arch Intern Med. 2011;171(21):1879-86.

12. Glickman SW, Lytle BL, Ou FS, Mears G, O'Brien S, Cairns CB, et al. Care processes associated with quicker door-in-door-out times for patients with STelevation-myocardial infarction requiring transfer: results from a statewide regionalization program. Circ Cardiovasc Qual Outcomes. 2011;4(4):382-8.

13. CCN. Cardiac Care Network of Ontario Ontario STEMI Bypass Protocol. 2015.

14. Kulynych J, Korn D. The effect of the new federal medical-privacy rule on research. N Engl J Med. 2002;346(3):201-4.

15. Tu JV, Willison DJ, Silver FL, Fang J, Richards JA, Laupacis A, et al. Impracticability of informed consent in the registry of the Canadian stroke network. N Engl J Med. 2004;350(14):1414-21.

16. Roffi M, Patrono C, Collet J, Mueller C, Valgimigli M, Andreotti F, et al. 2015 ESC guidelines for the management of acute coronary syndromes in patients presenting without persistent ST-segment elevation. Eur Heart J. 2016;37(3):267-315.

17. Chin $C T$, Chen AY, Wang TY, Alexander KP, Mathews R, Rumsfeld JS, et al. Risk adjustment for in-hospital mortality of contemporary patients with acute myocardial infarction: the acute coronary treatment and intervention outcomes network (ACTION) registry ${ }^{\circledR}$-get with the guidelines (GWTG) ${ }^{\mathrm{TM}}$ acute myocardial infarction mortality model and risk score. Am Heart J. 2011;161(1):113-22.

18. Cantor WJ, Fitchett D, Borgundvaag B, Ducas J, Heffernan M, Cohen EA, et al. Routine early angioplasty after fibrinolysis for acute myocardial infarction. N Engl J Med. 2009;360(26):2705-18.

19. Bøhmer E, Hoffmann P, Abdelnoor M, Arnesen $\mathrm{H}$, Halvorsen S. Efficacy and safety of immediate angioplasty versus ischemia-guided management after thrombolysis in acute myocardial infarction in areas with very long transfer distances. J Am Coll Cardiol. 2010;55(2):102-10.

20. Andersen HR, Nielsen TT, Vesterlund T, Grande P, Abildgaard U, Thayssen P, et al. Danish multicenter randomized study on fibrinolytic therapy versus acute coronary angioplasty in acute myocardial infarction: rationale and design of the danish trial in acute myocardial infarction-2 (DANAMI-2). Am Heart J. 2003;146(2):234-41.

21. Bhan V, Cantor WJ, Yan RT, Mehta SR, Morrison LJ, Heffernan M, et al. Efficacy of early invasive management post-fibrinolysis in men versus women with ST-elevation myocardial infarction: a subgroup analysis from trial of routine angioplasty and stenting after fibrinolysis to enhance reperfusion in acute myocardial infarction (TRANSFER-AMI). Am Heart J. 2012;164(3):343-50. 
22. Avezum A, Makdisse M, Spencer F, Gore JM, Fox KA, Montalescot G, et al. Impact of age on management and outcome of acute coronary syndrome: observations from the global registry of acute coronary events (GRACE). Am Heart J. 2005;149(1):67-73.

23. Glickman SW, Granger CB, Ou FS, O'Brien S, Lytle BL, Cairns CB, et al. Impact of a statewide ST-segment-elevation myocardial infarction regionalization program on treatment times for women, minorities, and the elderly. Circ Cardiovasc Qual Outcomes. 2010;3(5):514-21.

24. Davis MT, Dukelow A, McLeod S, Rodriguez S, Lewell M. The utility of the prehospital electrocardiogram. CJEM. 2011;13(06):372-7.

25. Bradley EH, Herrin J, Wang Y, Barton BA, Webster TR, Mattera JA, et al. Strategies for reducing the door-to-balloon time in acute myocardial infarction. N Engl J Med. 2006;355(22):2308-20.

Ready to submit your research? Choose BMC and benefit from:

- fast, convenient online submission

- thorough peer review by experienced researchers in your field

- rapid publication on acceptance

- support for research data, including large and complex data types

- gold Open Access which fosters wider collaboration and increased citations

- maximum visibility for your research: over $100 \mathrm{M}$ website views per year

At $\mathrm{BMC}$, research is always in progress.

Learn more biomedcentral.com/submissions 